Comment. Math. Helv. 74 (1999) 27-53

$0010-2571 / 99 / 010027-27 \$ 1.50+0.20 / 0$
(C) 1999 Birkhäuser Verlag, Basel

Commentarii Mathematici Helvetici

\title{
A factorization of the Conway polynomial
}

\author{
Jerome Levine
}

\begin{abstract}
It is shown that the Conway polynomial of a link is a product of two factors, the first of which is the Conway polynomial of a knot obtained by banding together the link components and the second is determined, via an explicit formula, by the $\tilde{\mu}$-invariants of the link. In particular we get a formula, in terms of the $\mu$-invariants, for the first non-zero coefficient of the Conway polynomial. A similar formula is obtained for the multi-variable Alexander-polynomial.
\end{abstract}

Mathematics Subject Classification (1991). 57M25.

Keywords. Conway polynomial, Alexander polynomial, link, knot, $\tilde{\mu}$-invariants.

\section{Introduction}

It is tempting to conjecture that there is some interesting relationship between the Conway polynomial $\nabla_{L}(z)$ of a link $L$ and $\nabla_{K}(z)$, where $K$ is a knot obtained by banding together the components of $L$. Obviously they cannot be equal since only terms of even or odd degree appear in $\nabla_{L}(z)$, according to whether $L$ has an odd or even number of components. Moreover there are many ways of choosing bands and one can easily see that the variety of knots one obtains can have very different polynomials. Nevertheless we will demonstrate that $\nabla_{L}(z)$ and $\nabla_{K}(z)$ have a very precise relationship in the form of a factorization:

$$
\nabla_{L}(z)=\nabla_{K}(z) \Gamma(z)
$$

where $\Gamma(z)$ is a power series in $z$ which depends on the choice of bands. More precisely the choice of bands can be viewed as the choice of a string link representation of $L$ and the coefficients of $\Gamma(z)$ are given by explicit formulae in terms of the Milnor $\bar{\mu}$-invariants of this string link. Thus it actually only depends on the I-equivalence class of $L$ (and the bands). From this point of view we see that the indeterminacy of the band choice is compensated by the notorious and familiar

Partially supported by NSF grant DMS-96-26639. This and other preprints of the author can be obtained on the WEB at the address http://www.math.brandeis.edu/Faculty/jlevine/. 
indeterminacy of the $\bar{\mu}$-invariants. However there is no indeterminacy in the first non-zero coefficient of $\nabla_{L}(z)$ and our results give a general formula for this coefficient in terms of the $\bar{\mu}$-invariants of $L$, generalizing the special cases obtained previously by Cochran, Hoste and myself.

We will also obtain an analogous factorization of the multivariable Alexander polynomial, depending on a choice of string link $S$ representing $L$ :

$$
\Delta_{L}\left(t_{1}, \cdots, t_{m}\right)=\theta\left(t_{1}, \cdots, t_{m}\right) \Gamma\left(t_{1}, \cdots, t_{m}\right)
$$

in which $\theta\left(t_{1}, \cdots, t_{m}\right)$ and $\Gamma\left(t_{1}, \cdots, t_{m}\right)$ are rational power series in $\left\{t_{i}-1\right\}$ with the properties that $\Gamma\left(t_{1}, \cdots, t_{m}\right)$ is given by an explicit formula in terms of the $\bar{\mu}$-invariants of $S$ and $\theta(1, \cdots, 1)=1$. However in this factorization we cannot tell if $\theta\left(t_{1}, \cdots, t_{m}\right)$ is a polynomial and, in any case, it lacks the geometric interpretation of the corresponding factor in the one-variable case. One interesting consequence though is a formula for the lowest degree terms in the Taylor expansion of $\Delta_{L}\left(t_{1}, \cdots, t_{m}\right)$ about $(1, \cdots, 1)$ in terms of the $\bar{\mu}$-invariants of $S$. These results are at least implicit in Traldi $[\mathrm{T}]$ and are closely related to work of Rozansky $[R]$. It is suggested by recent work of Habegger and Masbaum [HM] that such a factorization occurs for the general class of finite-type invariants of string links. ${ }^{1}$

Finally I would like to thank Stavros Garoufalidis for helpful discussions.

\subsection{Statement of results}

Suppose $L$ is an $m$-component oriented link in $\mathbb{R}^{3}$. We will assume $m \geq 2$ throughout this work. It is obvious that $L$ can be obtained by closing a string link $S$ - see Section 2.1 for the definitions. In fact there are many choices of different string links whose closure give $L$ if $m>1$. Now for any string link $S$ we will define, in Section 5, another form of closure which will produce a knot which we denote $K_{S}$. We will call $K_{S}$ the knot closure of $S$. In fact $K_{S}$ will be a band sum of the components of $L_{S}$ (the usual link closure of $S$ ). It is not hard to see that, for any knot $K$ obtained by band-summing of the components of a link $L$, there is some string link $S$ such that $L=L_{S}$ and $K=K_{S}$.

In [Mi] Milnor defined, for any oriented link $L$, an array of integer-valued invariants $\left\{\bar{\mu}_{i_{1}, \ldots, i_{k}}(L)\right\}$, for every choice of integers $1 \leq i_{r} \leq m$ and $k \geq 2$. The study of these invariants has always been hampered by a complicated selfreferencing indeterminacy in their definition. The simplest case when they are well-defined is formulated in the following recursive way: $\left\{\bar{\mu}_{i_{1}, \ldots, i_{k}}(L)\right\}$ is welldefined if $\left\{\bar{\mu}_{j_{1}, \ldots, j_{r}}(L)\right\}$ is well-defined and zero for any proper, order-preserving subset $\left\{j_{1}, \ldots, j_{r}\right\}$ of $\left\{i_{1}, \ldots, i_{k}\right\}$. So, for example, the $\bar{\mu}$-invariants of order $k$

\footnotetext{
1 A very recent preprint, The Gassner representation for string links by Paul Kirk, Charles Livingston and Zhenghan Wang, obtains some similar factorizations of the Alexander polynomials of a link with interesting interpretations of the factors.
} 
are well-defined if all those of order less than $k$ are well-defined and zero. When $k=2, \bar{\mu}_{i j}(L)$ is the linking number of the $i$-th and $j$-th components of $L$ if $i \neq j$.

The situation was improved by the introduction of string links. For a string link $S, \bar{\mu}_{i_{1}, \ldots, i_{k}}(S)$ is well-defined with no indeterminacy - the definition is recalled in Equation (2). Thus the indeterminacy of the $\bar{\mu}$-invariants of $L$ reflects the indeterminacy in the choice of a string link whose closure is $L$.

Our main result will be:

Theorem 1. Let $S$ be a string link, with closure $L_{S}$ and knot closure $K_{S}$. Then we have the following factorization of the Conway polynomial of $L_{S}$ :

$$
\nabla_{L_{S}}(z)=\nabla_{K_{S}}(z) \Gamma_{S}(z)
$$

where $\Gamma_{S}(z)$ is a power series given by the formula:

$$
\begin{gathered}
\Gamma_{S}(z)=(u+1)^{e / 2} \operatorname{det}\left(\lambda_{i j}(u)\right) \\
\text { with } z=u / \sqrt{u+1}, e=\left\{\begin{array}{ll}
0 & \text { if } m \text { is odd } \\
1 & \text { if } m \text { is even }
\end{array}\right. \text { and: } \\
\lambda_{i j}(u)=\sum_{r=0}^{\infty}\left(\sum_{i_{1}, \ldots, i_{r}} \bar{\mu}_{i_{1}, \ldots, i_{r}, j, i}(S)\right) u^{r+1} \quad \text { for } 1 \leq i, j \leq m-1
\end{gathered}
$$

Note that $\Gamma_{S}(z)$ is a rational function, i.e. it is a quotient of polynomials with integer coefficients.

Corollary 1.1. If the $\bar{\mu}$-invariants of $L$ vanish for order less than $k$, and so the $\bar{\mu}$-invariants of order $k$ are well-defined (i.e. have no indeterminacy), then $\nabla_{L}(z)$ is divisible by $z^{(k-1)(m-1)}$ and the coefficient of $z^{(k-1)(m-1)}$ is $\operatorname{det}\left(a_{i j}\right)$ where

$$
a_{i j}=\sum_{i_{1}, \ldots, i_{k-2}} \bar{\mu}_{i_{1}, \ldots, i_{k-2}, j, i}(L) \quad \text { for } 1 \leq i, j \leq m-1
$$

This generalizes results of Hoste $[\mathrm{H}]$, Cochran [C] (also see [T, Corollary 6.3]) and myself [L3].

Corollary 1.2. The first non-vanishing term (and its degree) of the Conway polynomial is an I-equivalence invariant.

This was first proved by Cochran [C]. I-equivalence is the relation generated by concordance and connected sum of a component with a local knot. A local knot is a knot lying in a ball disjoint from the link. 
To state the next results we use the following terminology. A polynomial or rational function $f(z)$ will be called norm-like if it can be written in the form $f(z)=h(t) h\left(t^{-1}\right)$ for some rational function $h(t)\left(z=t-t^{-1}\right)$. If $f(z)$ is normlike it must contain only even powers of $z$ and $f(0)$ must be square. But, for example, $z^{2}+a$ is norm-like if and only if $a=4$. Note that norm-like is not the same as being of the form $g(z) g(-z)$.

Corollary 1.3. If $L$ and $L^{\prime}$ are concordant links, then their Conway polynomials are related in the following way:

$$
\nabla_{L}(z) f(z)=\nabla_{L^{\prime}}(z) g(z)
$$

where $f, g$ are norm-like polynomials satisfying $f(0)=1=g(0)$.

This was first proved in $[\mathrm{C}]$; the analogous result for the multi-variable Alexander polynomial was first proved in $[\mathrm{Ka}]$ and $[\mathrm{N}]$.

We will also prove the following result about $\Gamma_{S}(z)$.

Proposition 1.4. Suppose that all the linking numbers of $L_{S}$ are zero. Then:

1. $\Gamma_{S}(z)$ has only even (resp., odd) powers of $z$ if $m$ is odd (resp., even).

2. $\Gamma_{S}(z)$ is divisible by $z^{2(m-1)}$. If $m$ is odd, then either $\Gamma_{S}(z)$ is divisible by $z^{2 m}$ or it is norm-like.

Corollary 1.5. If all the linking numbers of $L_{S}$ are zero then the value of $z^{-2(m-1)}$ $\Gamma_{S}(z)$ at $z=0$ is square.

This was recently proved in [L3].

For the multivariable Alexander polynomial we will prove an analogous, but less concrete, result. The Alexander polynomial $\Delta_{L}\left(t_{1}, \cdots, t_{m}\right)$ of an $m$-component $\operatorname{link} L$ is an element of the Laurent polynomial $\operatorname{ring} \mathbb{Z}\left[t_{1}, \cdots, t_{m}, t_{1}^{-1}, \cdots, t_{m}^{-1}\right]$, traditionally defined only up to multiplication by a unit in $\mathbb{Z}\left[t_{1}, \cdots, t_{m}, t_{1}^{-1}, \cdots, t_{m}^{-1}\right]$. We will make the substitutions $t_{i}=v_{i}+1$, which defines an imbedding of $\mathbb{Z}\left[t_{1}, \cdots\right.$, $\left.t_{m}, t_{1}^{-1}, \cdots, t_{m}^{-1}\right]$ into the power series ring $\mathbb{Z}\left[\left[v_{1}, \ldots, v_{m}\right]\right]$, and denote by $\Delta_{L}\left(v_{1}\right.$, $\left.\cdots, v_{m}\right)$ the image of $\Delta_{L}\left(t_{1}, \cdots, t_{m}\right)$. We will also need to make use of the element $\tau_{i}=\prod_{j=1}^{m} t_{j}^{l_{i j}} \in \mathbb{Z}\left[t_{1}, \cdots, t_{m}, t_{1}^{-1}, \cdots, t_{m}^{-1}\right] \subseteq \mathbb{Z}\left[\left[v_{1}, \ldots, v_{m}\right]\right]$, where $l_{i j}$ is the linking number of the $i$-th and $j$-th components of $L$ if $i \neq j$, and is defined by the equation $\sum_{j=1}^{m} l_{i j}=0$ for $i=j$.

Theorem 2. There exists a rational power series $\theta\left(v_{1}, \cdots, v_{m}\right)$ with constant term \pm 1 , such that

$$
\Delta_{L_{S}}\left(v_{1}, \cdots, v_{m}\right)=\Phi_{S}\left(v_{1}, \cdots, v_{m}\right) \theta\left(v_{1}, \cdots, v_{m}\right)
$$

where

$$
\Phi_{S}\left(v_{1}, \cdots, v_{m}\right)=\frac{1}{v_{m}} \operatorname{det}\left(a_{i j}\right)
$$


and $\left(a_{i j}\right)$ is the $(m-1) \times(m-1)$ matrix defined by

$$
a_{i j}=v_{i}\left(\sum_{i_{1}, \cdots, i_{r}} \bar{\mu}_{i_{1}, \ldots, i_{r}, j, i}(S) v_{i_{1}} \cdots v_{i_{r}}\right)-\left(\tau_{i}-1\right) \delta_{i j} \quad \text { for } 1 \leq i, j \leq m-1
$$

Corollary 1.6. If the $\bar{\mu}$-invariants of $L$ vanish for order less than $k$, and so the $\bar{\mu}$-invariants of order $k$ are well-defined (i.e. have no indeterminacy), then $\Delta_{L}\left(v_{1}, \cdots, v_{m}\right)$ has no terms of degree $<(k-1)(m-1)-1$ and the homogeneous part of $\Delta_{L}\left(v_{1}, \cdots, v_{m}\right)$ of degree $(k-1)(m-1)-1$ is $\operatorname{det}\left(a_{i j}\right) / v_{m}$, where, if $k \geq 3$ :

$$
a_{i j}=v_{i} \sum_{i_{1}, \cdots, i_{k-2}} \bar{\mu}_{i_{1}, \ldots, i_{k-2}, j, i}(L) v_{i_{1}} \cdots v_{i_{k-2}} \quad \text { for } 1 \leq i, j \leq m-1
$$

and, if $k=2$ :

$$
a_{i j}= \begin{cases}-l_{i j} v_{j} & \text { if } i \neq j \\ -\sum_{r \neq i} l_{i r} v_{r} & \text { if } i=j\end{cases}
$$

One more thing to mention is that the definition of $\bar{\mu}$-invariants used in this paper is slightly different from the classical definition by Milnor. However for algebraically split links $\left(\bar{\mu}_{i j}(S)=0\right.$ for all $\left.i \neq j\right)$ they coincide and, in general, there is a simple formula relating the two definitions. This is discussed below in Section 2.2.

\subsection{Outline of proof}

For the Conway polynomial we will produce factorizations $\nabla_{L}(z)=\nabla^{\prime}(z) \nabla^{\prime \prime}(z)$ from two different points of view. In Sections 3 and 4 we will examine the homology of the infinite cyclic cover of a string link and, in Theorem 3, produce a factorization in which $\nabla^{\prime}(0)=1$ and $\nabla^{\prime \prime}(z)=\Gamma_{S}(z)$. In Section 5 we will introduce the Seifert matrix of a string link and obtain another factorization, in equation (22), in which $\nabla^{\prime}(z)=\nabla_{K_{S}}(z)$ and $\nabla^{\prime \prime}(z)$ is defined from a Seifert matrix. The proof is completed by showing that these two factorizations are actually the same. This argument is carried out in Section 6 .

For Theorem 2 we only need carry out the homological argument. This is explained in Sections 3.2 and 4.3.

In Section 7 we prove Proposition 1.4 and in Section 8 we illustrate the factorization of $\nabla_{L}(z)$ given in Theorem 1 by a very simple example. 


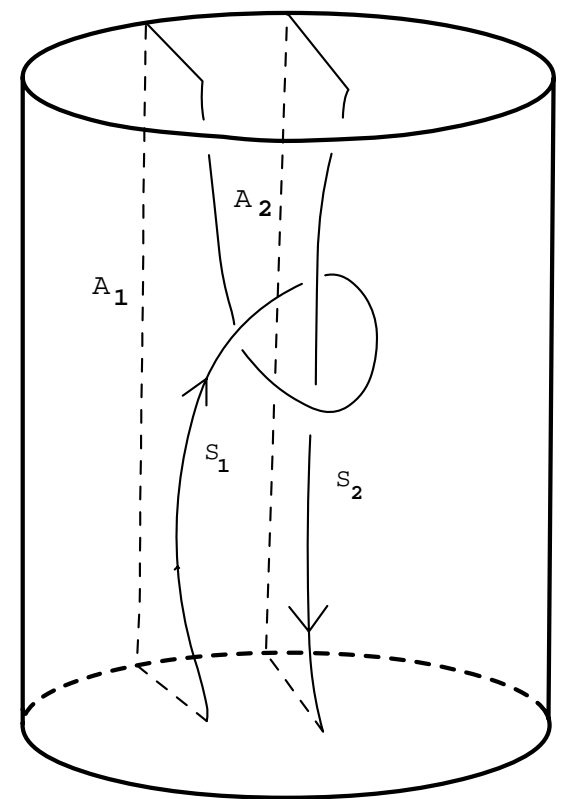

Figure 1.

Closure of a string link

\section{Preliminaries}

\subsection{String links}

We review the definition of the $\bar{\mu}$-invariants of a string link.

Recall that a string link $S$ is an ordered collection of disjoint oriented, properly imbedded $\operatorname{arcs} S_{1}, \ldots, S_{m}$ in $I \times D^{2}$. It will be convenient for our purposes to orient the strings in the following manner. The odd numbered strings $S_{i}$ will be directed from $\left(0, p_{i}\right)$ to $\left(1, p_{i}\right)$ and the even-numbered strings from $\left(1, p_{i}\right)$ to $\left(0, p_{i}\right)$, where $p_{1}, \ldots, p_{m}$ are prescribed distinct points in $D^{2}$. The closure $L_{S}$ of $S$ is the oriented link in $S^{3}$ whose components $L_{i}=S_{i} \cup A_{i}$, where $A_{i}$ are prescribed disjoint $\operatorname{arcs}$ in $\overline{S^{3}-I \times D^{2}}$ connecting $\left(1, p_{i}\right)$ and $\left(0, p_{i}\right)$, which meet $I \times D^{2}$ only at their endpoints. See Figure 1.

If $X=\left(I \times D^{2}\right)-S$ and $\pi=\pi_{1}(X)$, then $H_{1}(\pi)$ is free abelian of rank $m$ generated by meridian elements, and $H_{2}(\pi)=0$. The latter follows since we can choose a Wirtinger presentation for $\pi$ of deficiency $m$. We may choose canonical meridian elements $\mu_{1}, \ldots, \mu_{m} \in \pi$ represented by curves lying in $0 \times D^{2}$ (assuming the base point lies in $0 \times S^{1}$ ) so that the linking number of $\mu_{i}$ and $L_{i}$ is +1 . If $F$ is the free group on generators $x_{1}, \ldots, x_{m}$, then the map $F \rightarrow \pi$ defined by $x_{i} \rightarrow \mu_{i}$ induces isomorphisms $F / F_{q} \cong \pi / \pi_{q}$, for all $q$, and $\tilde{F} \rightarrow \tilde{\pi}$, where, for any group $G,\left\{G_{q}\right\}$ denotes the lower central series of $G^{-} G_{1}=G$ and $G_{q+1}=\left[G \cdot G_{q}\right]-$ and 
$\tilde{G}=\lim _{\longleftarrow} G_{i}$, the nilpotent completion. This follows from Stallings theorem [S]. We can also choose canonical longitudes $\lambda_{i} \in \pi$ with representative curves parallel to the components of $S$ and closed up in the boundary of $I \times D^{2}$ in a prescribed way, so that the total linking number of $\lambda_{i}$ is zero. The total linking number of any oriented closed curve $\alpha$ in $X$ is defined to be the sum of the linking numbers of $\alpha$ with all the components of $L_{S}$. This choice of $\lambda_{i}$ differs from the traditional choice in which the linking number of $\lambda_{i}$ and $L_{i}$ is required to be zero, but our choice will be more convenient for formulae. If the link is algebraically split, i.e. all the linking numbers of the components of $L_{S}$ are zero, then, of course, both choices are the same. In general, we have:

$$
\tilde{\lambda}_{i}=\mu_{i}^{l_{i}} \lambda_{i}
$$

where $\tilde{\lambda}_{i}$ denotes the traditional longitude and $l_{i}=\sum_{j \neq i} \bar{\mu}_{i j}(S)$.

\subsection{Magnus expansion and the $\bar{\mu}$-invariants}

In $[\mathrm{M}]$ Magnus defines an imbedding $\theta: F \rightarrow \mathbb{Z}\left[\left[u_{1}, \ldots, u_{m}\right]\right]$, where $\mathbb{Z}\left[\left[u_{1}, \ldots, u_{m}\right]\right]$ is the power-series ring in $m$ non-commuting variables $u_{1}, \ldots, u_{m}$, by defining $\theta\left(x_{i}\right)=1+u_{i}$ and extending this to a group homomorphism into the multiplicative group $1+\mathcal{I}$. $\mathcal{I}$ is the ideal in $\mathbb{Z}\left[\left[u_{1}, \ldots, u_{m}\right]\right]$ consisting of all series with zero constant term. Since $\theta\left(F_{q}\right) \subseteq 1+\mathcal{I}^{q}, \theta$ extends to define an imbedding $\tilde{F} \rightarrow \mathbb{Z}\left[\left[u_{1}, \ldots, u_{m}\right]\right]$. For a string link $S$ this induces an imbedding $\theta_{S}: \tilde{\pi} \rightarrow \mathbb{Z}\left[\left[u_{1}, \ldots, u_{m}\right]\right]$. We can then extend $\theta$ and $\theta_{S}$ to ring homomorphisms $\mathbb{Z} F \rightarrow \mathbb{Z}\left[\left[u_{1}, \ldots, u_{m}\right]\right]$ and $\mathbb{Z} \pi \rightarrow \mathbb{Z}\left[\left[u_{1}, \ldots, u_{m}\right]\right]$. Now, following the original concept in Milnor $[\mathrm{M}]$, but adapting to the string link context (see e.g. [L2]), we define the $\bar{\mu}$-invariants of $S$ by the formula

$$
\theta_{S}\left(\lambda_{i}\right)=1+\sum_{i_{1}, \ldots, i_{r}} \bar{\mu}_{i_{1}, \ldots, i_{r}, i}(S) u_{i_{1}} \cdots u_{i_{r}}
$$

The traditional $\bar{\mu}$-invariants (which we will denote $\tilde{\mu}_{i_{1}, \ldots, i_{r}, i}(S)$ ) are defined by the same formula, using $\tilde{\lambda}_{i}$ in place of $\lambda_{i}$. Using Equation (1) we can write down an explicit formula relating the two notions as follows. Let $1 \leq t \leq r$ be defined by the requirement that $i_{j}=i$, for $j<t$, and either $t=r$ or $i_{t} \neq i$. Then we have:

$$
\bar{\mu}_{i_{1}, \ldots, i_{r}, i}(S)=\sum_{s=1}^{t}\left(\begin{array}{c}
-l_{i} \\
s-1
\end{array}\right) \tilde{\mu}_{i_{s}, \ldots, i_{r}, i}
$$

Here $\left(\begin{array}{c}m \\ k\end{array}\right)$ denotes the usual binomial coefficient if $m$ is a positive integer and, in general, the coefficient of $x^{k}$ in the power series expansion of $(1+x)^{m}$, for any integer $m$.

If $L_{S}$ is the closure of $S$, then it is clear that $\tilde{\mu}_{i_{1}, \ldots, i_{r}}(S) \equiv \bar{\mu}_{i_{1}, \ldots, i_{r}}\left(L_{S}\right)$, modulo indeterminacy, where $\bar{\mu}_{i_{1}, \ldots, i_{r}}\left(L_{S}\right)$ denotes the classical $\bar{\mu}$-invariants of $L_{S}$ defined by Milnor in $[\mathrm{Mi}]$. 


\section{Homological invariants of string links}

\subsection{The longitudinal matrix}

Consider now the infinite cyclic cover $p: \tilde{X} \rightarrow X$ defined by the epimorphism $\pi \rightarrow \mathbf{Z}$ sending $\mu_{i} \rightarrow t$, where $\mathbf{Z}$ is the infinite cyclic multiplicative group with generator $t$. We consider the relative homology group $H_{1}(\tilde{X}, \tilde{*})$, where $*$ is the base-point of $X$ and $\tilde{*}=p^{-1}(*)$. This is a module over the Laurent polynomial ring $\mathbb{Z}\left[t, t^{-1}\right]$. Consider the multiplicative subset $\Sigma \subseteq \mathbb{Z}\left[t, t^{-1}\right]$ consisting of all $f(t)$ such that $f(1)=1$, and the localization $\mathbb{Z}\left[t, t^{-1}\right]_{\Sigma}$ of $\mathbb{Z}\left[t, t^{-1}\right]$ consisting of all quotients of elements of $\mathbb{Z}\left[t, t^{-1}\right]$ by elements of $\Sigma$. We could, alternatively, consider the completion of $\mathbb{Z}\left[t, t^{-1}\right]$. Let $\mathbb{Z}[[u]]$ denote the ring of power series in the variable $u$. Then we can define a ring homomorphism $\tilde{\theta}: \mathbb{Z}\left[t, t^{-1}\right] \rightarrow \mathbb{Z}[[u]]$ by $\tilde{\theta}(t)=1+u$. Since $\tilde{\theta}(\Sigma) \subseteq$ units of $\mathbb{Z}[[u]], \tilde{\theta}$ extends to an imbedding $\mathbb{Z}\left[t, t^{-1}\right]_{\Sigma} \rightarrow \mathbb{Z}[[u]]$ which we still denote by $\tilde{\theta}$.

Lemma 3.1. $H_{1}(\tilde{X}, \tilde{*})_{\Sigma}$ is a free $\mathbb{Z}\left[t, t^{-1}\right]_{\Sigma}$-module with basis $\tilde{\mu}_{1}, \ldots, \tilde{\mu}_{m}$, where $\tilde{\mu}_{i}$ is represented by a lift of $\mu_{i}$ to a curve in $\tilde{X}$ starting at some prescribed basepoint $\hat{*} \in \tilde{*}$.

For a proof see [L1]. This lemma will also follow from the argument given below in Section 6.1.

Now suppose we define $\tilde{\lambda}_{i} \in H_{1}(\tilde{X}, \tilde{*})$ to be the class represented by the lift of $\lambda_{i}$ to a path beginning at $\hat{*}$. Then we can write, in $H_{1}(\tilde{X}, \tilde{*})_{\Sigma}$ :

$$
\tilde{\lambda}_{i}=\sum_{j=1}^{m} c_{i j}^{S} \tilde{\mu}_{j}, \quad 1 \leq i \leq m
$$

We will show how the $c_{i j}^{S}$ are determined by the $\bar{\mu}$-invariants of $S$.

Lemma 3.2. If we write

$$
\tilde{\theta}\left(c_{i j}^{S}\right)=\sum_{k=0}^{\infty} c_{i j k}(S) u^{k}
$$

then

$$
c_{i j k}(S)=\sum_{i_{1}, \ldots, i_{k}} \bar{\mu}_{i_{1}, \ldots, i_{k}, j, i}(S)
$$

Proof. The relation between the image of $\lambda_{i}$ under the mapping $\pi \rightarrow \tilde{\pi} \cong \tilde{F}$ and $\tilde{\lambda}_{i} \in H_{1}(\tilde{X}, \tilde{*})_{\Sigma} \cong \mathbb{Z}\left[t, t^{-1}\right]_{\Sigma}\left[\tilde{\mu}_{1}, \ldots, \tilde{\mu}_{m}\right]$ is well-understood, on the purely algebraic level, to be given by the Fox free differential calculus as follows. If $g \in F$, 
then consider $g-1 \in I F \subseteq \mathbb{Z}[F]$, where $I F$ is the augmentation ideal in $\mathbb{Z}[F]$. IF, regarded as a left $\mathbb{Z}[F]$-module, is freely generated by the elements $x_{1}-1, \ldots, x_{m}-$ 1 and so we can write $g-1=\sum_{i} a_{i}\left(x_{i}-1\right)$. Let $\eta: \mathbb{Z}[F] \rightarrow \mathbb{Z}\left[t, t^{-1}\right]$ be defined by $\eta\left(x_{i}\right)=t$. Let $\tilde{g}$ denote the element $\sum_{i} \eta\left(a_{i}\right) X_{i} \in \mathbb{Z}\left[t, t^{-1}\right]\left[X_{1}, \ldots, X_{m}\right]$, the free $\mathbb{Z}\left[t, t^{-1}\right]$-module with basis $X_{1}, \ldots, X_{m}$. Then $\delta(g)=\tilde{g}$ defines a function $\delta: F \rightarrow \mathbb{Z}\left[t, t^{-1}\right]\left[X_{1}, \ldots, X_{m}\right]$, which is additive and has the property $\delta(g h)=$ $\eta(g) \delta(h)+\delta(g)$. Also note that $\delta\left(F_{q+1}\right) \subseteq I^{q} \mathbb{Z}\left[t, t^{-1}\right]\left[X_{1}, \ldots, X_{m}\right]$, where $I$ is the augmentation ideal of $\mathbb{Z}\left[t, t^{-1}\right]$. It follows that $\delta$ induces a function $F / F_{q+1} \rightarrow$ $\left(\mathbb{Z}\left[t, t^{-1}\right] / I^{q}\right)\left[X_{1}, \ldots, X_{m}\right]$.

Now if $\lambda_{i q}$ is the reduction of $\lambda_{i}$ into $\pi / \pi_{q+1} \cong F / F_{q+1}$, then $\delta\left(\lambda_{i q}\right)$ is the reduction of $\tilde{\lambda}_{i}$ into $H_{1}(\tilde{X}, \tilde{*})_{\Sigma} / I^{q} H_{1}(\tilde{X}, \tilde{*})_{\Sigma} \cong\left(\mathbb{Z}\left[t, t^{-1}\right] / I^{q}\right)\left[X_{1}, \ldots, X_{m}\right]$, where $\tilde{\mu}_{i} \rightarrow X_{i}$.

Let us now write

$$
\lambda_{i q}-1=\sum_{j} a_{i j}\left(x_{j}-1\right)
$$

where the $a_{i j}$ are well-defined $\bmod (I F)^{q}$. Then, by the discussion above, we have

$$
\tilde{\lambda}_{i} \equiv \sum_{j} \eta\left(a_{i j}\right) \tilde{\mu}_{j} \quad \bmod I^{q}
$$

and so $c_{i j}^{S} \equiv \eta\left(a_{i j}\right) \bmod \mathcal{I}^{q}$. From this we have

$$
\tilde{\theta}\left(c_{i j}^{S}\right) \equiv \tilde{\eta}\left(\theta\left(a_{i j}\right)\right) \quad \bmod u^{q}
$$

where $\tilde{\eta}: \mathbb{Z}\left[\left[u_{1}, \ldots, u_{m}\right]\right] \rightarrow \mathbb{Z}[[u]]$ is defined by $\tilde{\eta}\left(u_{i}\right)=u$.

On the other hand if we apply $\theta_{S}$ to equation (6) we get

$$
\theta_{S}\left(\lambda_{i q}\right) \equiv 1+\sum_{j} \theta\left(a_{i j}\right) u_{j} \quad \bmod I^{q+1}
$$

Comparing this with equation (2) we have

$$
\sum_{j} \theta\left(a_{i j}\right) u_{j} \equiv \sum_{i_{1}, \ldots, i_{r}} \bar{\mu}_{i_{1}, \ldots, i_{r}, i}(S) u_{i_{1}} \cdots u_{i_{r}} \quad \bmod \mathcal{I}^{q+1}
$$

and from this we see that

$$
\theta\left(a_{i j}\right) \equiv \sum_{i_{1}, \ldots, i_{r}} \bar{\mu}_{i_{1}, \ldots, i_{r}, j, i}(S) u_{i_{1}} \cdots u_{i_{r}} \quad \bmod \mathcal{I}^{q}
$$

Finally we combine equations (7) and (8) to get

$$
\tilde{\theta}\left(c_{i j}^{S}\right) \equiv \sum_{i_{1}, \ldots, i_{r}} \bar{\mu}_{i_{1}, \ldots, i_{r}, j, i} u^{r} \quad \bmod u^{q}
$$

Since we can take $q$ as large as we want, the proof is complete. 


\subsection{The universal abelian cover}

The considerations of Section 3.1 extend readily to the universal abelian cover of $X$. The results essentially correspond to those obtained by Traldi in $[\mathrm{T}]$ where the role of string link is replaced by a choice of link projection. We will omit proofs since they are identical to the arguments in Section 3.1. In fact the results in Sections 3 and 4 on the infinite cyclic covering and the one-variable polynomial are consequences of the analogous results for the universal abelian covering and the multivariable polynomial, but we will need the details of the argument in the one-variable case for the arguments in Sections 5 and after.

The universal abelian covering $p: \hat{X} \rightarrow X$ is defined by the epimorphism $\pi \rightarrow \mathbf{Z}^{m}$ sending $\mu_{i} \rightarrow t_{i}$, where $\mathbf{Z}^{m}$ is the free abelian (multiplicative) group with generators $t_{1}, \cdots t_{m}$. We consider the relative homology group $H_{1}(\hat{X}, \hat{*})$, where $*$ is the base-point of $X$ and $\hat{*}=p^{-1}(*)$. This is a module over the Laurent polynomial ring $\mathbb{Z}\left[t_{1}, \cdots, t_{m}, t_{1}^{-1}, \cdots, t_{m}^{-1}\right]$. Consider the multiplicative subset $\Sigma \subseteq$ $\mathbb{Z}\left[t_{1}, \cdots, t_{m}, t_{1}^{-1}, \cdots, t_{m}^{-1}\right]$ consisting of all $f\left(t_{1}, \cdots, t_{m}\right)$ such that $\left.f(1, \cdots, 1)\right)=$ 1 , and the localization $\mathbb{Z}\left[t_{1}, \cdots, t_{m}, t_{1}^{-1}, \cdots, t_{m}^{-1}\right]_{\Sigma}$ of $\mathbb{Z}\left[t_{1}, \cdots, t_{m}, t_{1}^{-1}, \cdots, t_{m}^{-1}\right]$ consisting of all quotients of elements of $\mathbb{Z}\left[t_{1}, \cdots, t_{m}, t_{1}^{-1}, \cdots, t_{m}^{-1}\right]$ by elements of $\Sigma$. We also consider the completion of $\mathbb{Z}\left[t_{1}, \cdots, t_{m}, t_{1}^{-1}, \cdots, t_{m}^{-1}\right]$. Let $\mathbb{Z}\left[\left[v_{1}, \ldots, v_{m}\right]\right]$ denote the ring of power series in the variable $v_{1}, \cdots, v_{m}$. Then we can define a ring homomorphism $\hat{\theta}: \mathbb{Z}\left[t_{1}, \cdots, t_{m}, t_{1}^{-1}, \cdots, t_{m}^{-1}\right] \rightarrow \mathbb{Z}\left[\left[v_{1}, \ldots, v_{m}\right]\right]$ by $\hat{\theta}\left(t_{i}\right)=1+v_{i}$. Since $\hat{\theta}(\Sigma) \subseteq$ units of $\mathbb{Z}\left[\left[v_{1}, \ldots, v_{m}\right]\right], \hat{\theta}$ extends to an imbedding $\mathbb{Z}\left[t_{1}, \cdots, t_{m}, t_{1}^{-1}, \cdots, t_{m}^{-1}\right]_{\Sigma} \rightarrow \mathbb{Z}\left[\left[v_{1}, \ldots, v_{m}\right]\right]$ which we still denote by $\hat{\theta}$.

Lemma 3.3. $H_{1}(\hat{X}, \hat{*})_{\Sigma}$ is a free $\mathbb{Z}\left[t_{1}, \cdots, t_{m}, t_{1}^{-1}, \cdots, t_{m}^{-1}\right]_{\Sigma}$-module with basis $\hat{\mu}_{1}, \ldots, \hat{\mu}_{m}$, where $\hat{\mu}_{i}$ is represented by a lift of $\mu_{i}$ to a curve in $\hat{X}$ starting at some prescribed base-point in $\hat{*}$.

We define $\hat{\lambda}_{i} \in H_{1}(\hat{X}, \hat{*})$ to be the class represented by the lift of $\lambda_{i}$ to a path beginning at the chosen base point. Then we can write, in $H_{1}(\hat{X}, \hat{*})_{\Sigma}$ :

$$
\hat{\lambda}_{i}=\sum_{j=1}^{m} \hat{c}_{i j}^{S} \hat{\mu}_{j}, \quad 1 \leq i \leq m
$$

We now have

Lemma 3.4.

$$
\hat{\theta}\left(\hat{c}_{i j}^{S}\right)=\sum_{i_{1}, \ldots, i_{k}} \bar{\mu}_{i_{1}, \ldots, i_{k}, j, i}(S) v_{i_{1}} \cdots v_{i_{k}}
$$




\subsection{Relations in the longitudinal matrix}

We now point out that the matrices $\left(c_{i j}^{S}\right)$ and $\left(\hat{c}_{i j}^{S}\right)$ are degenerate.

\section{Lemma 3.5.}

$$
\sum_{i=1}^{m} c_{i j}^{S}=0=\sum_{j=1}^{m} c_{i j}^{S}
$$

Proof. First recall that the $\left\{\lambda_{i}\right\}$ satisfy the relation which, with our orientation convention, reads:

$$
\left(\lambda_{1}^{-1} \mu_{1}^{-1} \lambda_{1}\right)\left(\lambda_{2} \mu_{2} \lambda_{2}^{-1}\right)\left(\lambda_{3}^{-1} \mu_{3}^{-1} \lambda_{3}\right) \cdots=\mu_{1}^{-1} \mu_{2} \mu_{3}^{-1} \cdots
$$

This is apparent since both sides are represented by $0 \times S^{1} \subseteq X$. If we apply the free differential calculus to this relation we obtain the following relation in $H_{1}(\tilde{X}, \tilde{*})$ :

$$
\sum_{i \text { odd }} t^{-l_{i}}\left(\left(t^{-1}-1\right) \tilde{\lambda}_{i}-t^{-1} \tilde{\mu}_{i}\right)+\sum_{i \text { even }}\left(\left(t^{-1}-1\right) \tilde{\lambda}_{i}+t^{-1} \tilde{\mu}_{i}\right)=\sum_{i=1}^{m}(-1)^{i} t^{-1} \tilde{\mu}_{i}
$$

where $l_{i}$ is the total linking number of $\lambda_{i}$. Since we have chosen $\lambda_{i}$ so that $l_{i}=0$, this equation becomes $\left(t^{-1}-1\right) \sum_{i=1}^{m} \tilde{\lambda}_{i}=0$. Since we are in a free module this becomes, simply, $\sum_{i=1}^{m} \tilde{\lambda}_{i}=0$, which proves the first equality.

Consider the boundary operator $\partial: H_{1}(\tilde{X}, \tilde{*}) \rightarrow H_{0}(\tilde{*}) \cong \mathbb{Z}\left[t, t^{-1}\right]$ from the homology sequence of $(\tilde{X}, \tilde{*})$. Then $\partial\left(\tilde{\mu}_{i}\right)=t-1$ and $\partial\left(\tilde{\lambda}_{i}\right)=t^{l_{i}}-1$. Thus we obtain the equality

$$
t^{l_{i}}-1=\partial\left(\tilde{\lambda}_{i}\right)=\sum_{j} c_{i j}^{S} \partial\left(\tilde{\mu}_{j}\right)=(t-1) \sum_{j} c_{i j}^{S}
$$

Since $l_{i}=0$ and $t-1$ is a non-zero divisor, we obtain the second inequality.

The degeneracy for $\left(\hat{c}_{i j}^{S}\right)$ is somewhat more complicated, but the argument is identical to that for Lemma 3.5.

Lemma 3.6. 1. If $\tau_{i}$ is as defined above in Section 1.1, then

$$
\tau_{i}-1=\sum_{j=1}^{m}\left(t_{j}-1\right) \hat{c}_{i j}^{S} .
$$

2.

$$
a_{j}\left(\tau_{j}-1\right)=\sum_{i=1}^{m} a_{i}\left(t_{i}-1\right) \hat{c}_{i j}^{S}
$$


where $a_{i}=b_{i} \cdot \prod_{r \text { odd }<i} t_{r}^{-1} \cdot \prod_{r \text { even }<i} t_{r}$ and $b_{i}=\left\{\begin{array}{ll}1 & \text { if } i \text { even } \\ \tau_{i}^{-1} t_{i}^{-1} & \text { if } i \text { odd }\end{array}\right.$.

Proof.Consider the boundary operator

$$
\partial: H_{1}(\hat{X}, \hat{*}) \rightarrow H_{0}(\hat{*}) \cong \mathbb{Z}\left[t_{1}, \cdots, t_{m}, t_{1}^{-1}, \cdots, t_{m}^{-1}\right]
$$

from the homology sequence of $(\hat{X}, \hat{*})$. Then $\partial\left(\hat{\mu}_{i}\right)=t_{i}-1$ and $\partial\left(\hat{\lambda}_{i}\right)=\tau_{i}-1$ and the first equality follows.

The second equality is proved by applying the Fox differential calculus to Equation (12) as in the proof of Lemma 3.5.

\section{First results on the Alexander polynomial}

\subsection{A presentation of the Alexander module}

Let $Y$ denote the complement of $L_{S}$, the closure of $S$. Then $Y=X \cup X_{0}$, where $X_{0}$ is the complement of the trivial $m$-component string link. One sees immediately that $\pi_{1}(Y) \cong \pi_{1}(X) /<\left[\mu_{i}, \lambda_{i}\right]>$ and, therefore, that:

$$
H_{1}(\tilde{Y}, \tilde{*}) \cong H_{1}(\tilde{X}, \tilde{*}) / M
$$

where $M$ is the submodule generated by the elements $(1-t) \tilde{\lambda}_{i}-\left(1-t^{l_{i}}\right) \tilde{\mu}_{i}$. Since $l_{i}=0$ we conclude that $H_{1}(\tilde{Y}, \tilde{*})$ has a presentation with $m$ generators $\left\{\tilde{\mu}_{i}\right\}$ and $m$ relators $\left\{(1-t) \sum_{j} c_{i j}^{S} \tilde{\mu}_{j}\right\}$.

\subsection{The Alexander polynomial}

Recall the definition of the Alexander polynomial $\Delta_{L}(t)$ of a link $L$ as a generator of the order ideal of $H_{1}(\tilde{Y})$, where $Y$ is the complement of $L$. It follows from the homology exact sequence of $(\tilde{Y} . \tilde{*})$ that $H_{1}(\tilde{Y}, \tilde{*}) \cong H_{1}(\tilde{Y}) \oplus \mathbb{Z}\left[t, t^{-1}\right]$ and so the order ideal of $H_{1}(\tilde{Y})$ is the same as the ideal generated by the $(k \times k)$ minors of the matrix of a presentation of $H_{1}(\tilde{Y}, \tilde{*})$ with $k+1$ generators. Thus we can conclude that the image of $\Delta_{L_{S}}(t)$ in $\mathbb{Z}\left[t, t^{-1}\right]_{\Sigma}$ is a generator of the ideal generated by the $(m-1) \times(m-1)$ minors of the matrix $(t-1) c_{i j}^{S}$. Since, by Lemma 3.5, the sum of the rows and the sum of the columns is zero, we have:

Lemma 4.1. The image of $\Delta_{L_{S}}(t)$ in $\mathbb{Z}\left[t, t^{-1}\right]_{\Sigma}$ is, up to multiplication by an element of $\Sigma,(t-1)^{m-1} \operatorname{det} C^{S}$, where $C^{S}$ is the $(m-1) \times(m-1)$ matrix whose entries are $\left\{c_{i j}^{S}, 1 \leq i, j \leq m-1\right\}$.

Now define $\Delta_{L}(u)=\tilde{\theta}\left(\Delta_{L}(t)\right) \in \mathbb{Z}[[u]]$. Then, as a consequence of Lemmas 3.2 and 4.1, we have: 
Theroem 3. There exists a rational power series $\theta(u)$ with constant term \pm 1 such that

$$
\Delta_{L_{S}}(u)=\Phi_{S}(u) \theta(u)
$$

where $\Phi_{S}(u)=u^{m-1} \operatorname{det}\left(a_{i j}\right)$, and $\left(a_{i j}\right)$ is the $(m-1) \times(m-1)$-matrix defined by:

$$
a_{i j}=\sum_{1 \leq i_{1}, \ldots, i_{r} \leq m} \bar{\mu}_{i_{1}, \ldots, \subset_{r}, j, i}(S) u^{r} \quad \text { for } 1 \leq i, j \leq m-1
$$

A rational power series is one which is the expansion of a quotient of polynomials in $u$. For example, the elements of $\operatorname{Im} \tilde{\theta}$ are rational. See [T1] for a result about the multivariable Conway polynomial which is similar to Theorem 3 .

From this theorem we can see, in particular, that the first non-vanishing coefficient of $\Delta_{L_{S}}(u)$ is, up to sign, equal to the first non-vanishing coefficient of $\Phi_{S}(u)$. In particular we have proved Corollary 1.1, for $\Delta_{L_{S}}(u)$ rather than the Conway polynomial, up to sign.

We can also obtain Corollary 1.2 for $\Delta_{L_{S}}(u)$, up to sign. Suppose $L_{1}$ and $L_{2}$ are concordant. We may assume, by a theorem of Tristram [Tr], that $L_{2}$ is obtained from $L_{1}$ by a ribbon move. In other words, for some trivial link $T$ in a ball $B$ disjoint from $L_{1}$, we obtain $L_{2}$ by band-summing each component of $T$ to some component of $L_{1}$. Now we can lift $L_{1}$ to a string link $S_{1}$ by choosing a so-called $d$-base (see Habegger-Lin $[\mathrm{HaL}]$ ), i.e. an imbedded 2-disk which meets each component of $L_{1}$ in a single point. Clearly we can choose a d-base which is disjoint from $B$ and the bands used to obtain $L_{2}$. So the same d-base can be used for $L_{2}$ and lifts $L_{2}$ to a string link $S_{2}$ concordant to $S_{1}$. Since the $\bar{\mu}$-invariants of a string link are concordance invariants (see $[S]), \Phi_{S_{1}}(u)=\Phi_{S_{2}}(u)$. Thus it follows from Theorem 3 that the first non-vanishing coefficients of $\Delta_{L_{i}}(u)$ are the same (up to sign). Finally, connect sum of a link with a local knot clearly multiplies the Alexander polynomial of the link by the Alexander polynomial of the knot.

\subsection{The multivariable Alexander polynomial}

The same argument as in Section 4.1 shows that $H_{1}(\hat{Y}, \hat{*})$ has a presentation with generators $\hat{\mu}_{1}, \cdots, \hat{\mu}_{m}$ and relations

$$
\left(\tau_{i}-1\right) \hat{\mu}_{i}=\left(t_{i}-1\right) \hat{\lambda}_{i}=\sum_{j=1}^{m}\left(t_{i}-1\right) \hat{c}_{i j}^{S} \hat{\mu}_{j}
$$

The usual definition of the multivariable Alexander polynomial

$\Delta_{L}\left(t_{1}, \cdots, t_{m}\right)$ is the greatest common divisor of the ideal $E$ generated by the $(k \times$ $k)$-minors of a presentation matrix with $(k+1)$ generators of the $\mathbb{Z}\left[t_{1}, \cdots, t_{m}, t_{1}^{-1}\right.$, $\left.\cdots, t_{m}^{-1}\right]$-module $H_{1}(\hat{Y}, \hat{*})$. We have the presentation matrix

$$
P=\left(\left(t_{i}-1\right) \hat{c}_{i j}^{S}-\delta_{i j}\left(\tau_{i}-1\right)\right)
$$


for $H_{1}(\hat{Y}, \hat{*})_{\Sigma}$ and so $E_{\Sigma}$ is generated by the $(m-1) \times(m-1)$-minors of $P$. By Lemma 3.6(2) the sum of unit multiples of the rows is zero. By Lemma 3.6(1) we obtain the formula

$$
\sum_{j}\left(t_{j}-1\right) P_{j}=0
$$

where $P_{j}$ is the $j$-th column of $P$. From (16) we can conclude that any $(m-$ $1) \times(m-1)$-minor $\Delta_{j}$ of $P$ obtained by omitting any row and the $j$-th column is divisible by $\left(t_{j}-1\right)$ and is independent (up to unit multiple) of the particular row which is omitted. Then it follows from (16) that $\Delta_{L_{S}}\left(t_{1}, \cdots t_{m}\right)$ is a unit multiple of $\Delta_{j} /\left(t_{j}-1\right)$ in $\mathbb{Z}\left[t_{1}, \cdots, t_{m}, t_{1}^{-1}, \cdots, t_{m}^{-1}\right]_{\Sigma}$. Choosing $j=m$ and applying Lemma 3.4 proves Theorem 2 .

The remainder of this paper will focus on the one-variable polynomial and, in particular, be devoted to sharpening Theorem 3 to obtain Theorem 1.

\section{The Conway polynomial}

We recall the definition of the Conway polynomial of a link $L$ in $\mathbb{R}^{3}$ (see, for example, [Le3]). Let $V$ be a Seifert surface for $L$, i.e. $V \subseteq \mathbb{R}^{3}$ is an oriented surface with $\partial V=L$. We define the Seifert pairing $\sigma: H_{1}(V) \times H_{1}(V) \rightarrow \mathbb{Z}$ by $\sigma(\alpha, \beta)=\lambda\left(j_{*} \alpha, \beta\right)$ where $j: V \rightarrow \mathbb{R}^{3}-V$ is defined by a push in the positive normal direction and $\lambda: H_{1}\left(\mathbb{R}^{3}-V\right) \times H_{1}(V) \rightarrow \mathbb{Z}$ is the linking pairing, which is non-singular by Alexander duality. Let $A$ be a matrix representing $\sigma$ with respect to a basis of $H_{1}(V)$. Then define the potential function $\Omega_{L}(t)=\operatorname{det}\left(t A-t^{-1} A^{t}\right)$. This Laurent polynomial depends only on $L$ and $\Omega_{L}(t)$ is a polynomial in $t-t^{-1}$. Thus we may define the Conway polynomial $\nabla_{L}(z)$ by the equation $\nabla_{L}\left(t-t^{-1}\right)=$ $\Omega_{L}(t)$.

Now suppose we are given a string link $S=\left\{S_{i}\right\}$. We have already discussed the link closure $L_{S}$ in Section 2.1; now we associate an oriented knot $K_{S}$ to $S$ by closing it in the following different manner, as indicated in Figure 2.

Insert bands $B_{i} \approx I \times I$, for $1 \leq i \leq m-1$, into $I \times S^{1}$ so that $B_{i}$ connects $S_{i}$ to $S_{i+1}$ and $B_{i} \cap S_{i} \approx I \times 0$ and $B_{i} \cap S_{i+1} \approx I \times 1$. We also arrange them so that $B_{i+1}$ is below $B_{i}$. Then $K_{S}$ is obtained from $L_{S} \cup \bigcup_{i} B_{i}$ by removing the part of each $B_{i}$ corresponding to $(0,1) \times I$ and orienting it consistent with the orientation of $L_{S}$. Now choose a Seifert surface $W$ for $K_{S}$ in $I \times D^{2}$ so that $W \cap \partial\left(I \times D^{2}\right)=K_{S} \cap \partial\left(I \times D^{2}\right)$. By the simple modification of merely adjoining the bands $\left\{B_{i}\right\}$ to $W$, we can convert $W$ into a Seifert surface $V$ for $L_{S}$ (see Figure 3).

The Seifert pairings and matrices of $W$ and $V$ are closely related. It is clear that the inclusion $W \subseteq V$ induces an isomorphism:

$$
H_{1}(V) \cong H_{1}(W) \oplus \mathbb{Z}^{m-1}
$$

The $\mathbb{Z}^{m-1}$ summand has, as basis, the classes represented by any $m-1$ of the components $\left\{L_{i}\right\}$ of $L_{S}$. We will usually choose $L_{1}, \ldots, L_{m-1}$. The Seifert pairing 


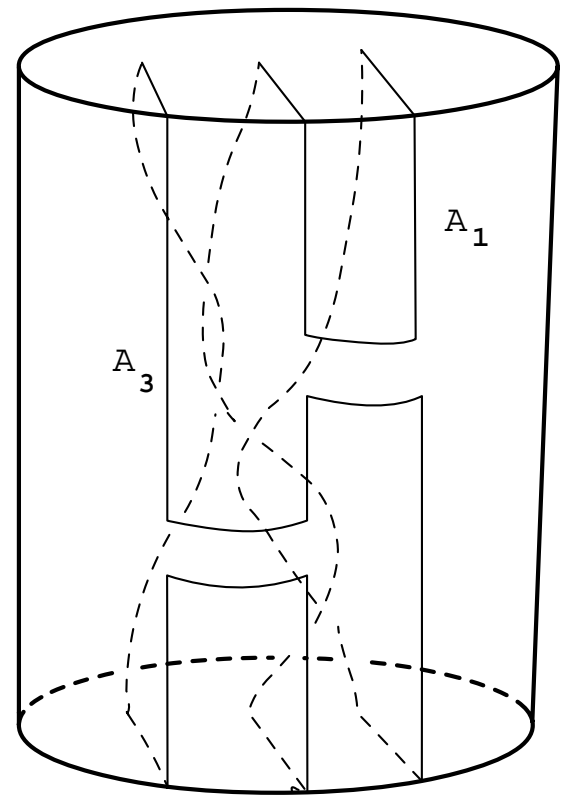

Figure 2 .

The knot closure of a string link

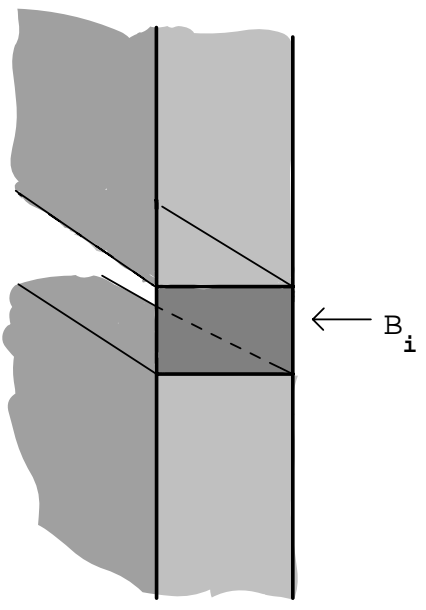

Figure 3.

Adjoining a band to a Seifert surface

of $V$, when restricted to $W$, obviously coincides with that of $W$. Therefore if $A$ is the Seifert matrix of $W$, with respect to some basis of $H_{1}(W)$, then the Seifert 
matrix $\bar{A}$ of $V$ will be of the form:

$$
\bar{A}=\left(\begin{array}{cc}
A & B^{t} \\
B & \Lambda
\end{array}\right)
$$

where $M^{t}$ denotes the transpose of the matrix $M$ and $\Lambda=\left(l_{i j}\right), 1 \leq i, j \leq m-1$ where $($ for $1 \leq i, j \leq m)$

$$
l_{i j}= \begin{cases}\text { linking number of } L_{i} \text { and } L_{j} & \text { if } i \neq j \\ -\sum_{r \neq i} l_{i r} & \text { if } i=j\end{cases}
$$

Note that the parallel push-off of each $L_{i}$ has total linking number 0 . The Conway polynomials of $K_{S}$ and $L_{S}$ are given by:

$$
\begin{aligned}
& \nabla_{K_{S}}\left(s-s^{-1}\right)=\operatorname{det}\left(s A-s^{-1} A^{t}\right) \\
& \nabla_{L_{S}}\left(s-s^{-1}\right)=\operatorname{det}\left(s \bar{A}-s^{-1} \bar{A}^{t}\right)
\end{aligned}
$$

Since $E=A-A^{t}$ is unimodular, it follows that $\nabla_{K_{S}}(0)=1$, as usual. Setting $z=s-s^{-1}$, the Conway polynomials lie in the polynomial $\operatorname{ring} \mathbb{Z}[z] \subseteq \mathbb{Z}\left[s, s^{-1}\right]$. Let $\Sigma^{\prime}$ denote the multiplicative subset of $\mathbb{Z}\left[s, s^{-1}\right]$ consisting of all $f(s)$ satisfying $f(1)= \pm f(-1)= \pm 1$. Then, over the localized ring $\mathbb{Z}\left[s, s^{-1}\right]_{\Sigma^{\prime}}$, the matrix $\mathcal{S}=s A-s^{-1} A^{t}=z A-s^{-1} E$ is invertible. We can, therefore, by elementary row operations in $\mathbb{Z}\left[s, s^{-1}\right]_{\Sigma^{\prime}}$ convert $s \bar{A}-s^{-1} \bar{A}^{t}$ into

$$
\overline{\mathcal{S}}=\left(\begin{array}{cc}
\mathcal{S} & B^{t} \\
0 & z \Lambda-z^{2} B \mathcal{S}^{-1} B^{t}
\end{array}\right)
$$

From this we obtain

$$
\nabla_{L_{S}}(z)=\nabla_{K_{S}}(z) \Gamma_{S}(z)
$$

where

$$
\Gamma_{S}(z)=\operatorname{det}\left(z \Lambda-z^{2} B \mathcal{S}^{-1} B^{t}\right)
$$

Note that, although $\Gamma_{S}$ is, by its definition, an element of $\mathbb{Z}\left[s, s^{-1}\right]_{\Sigma^{\prime}}$, it follows from (22) that it is a quotient of polynomials in $z$ or, alternatively, a power series in $z$.

\section{The Seifert matrix and the $\bar{\mu}$-invariants}

\subsection{Homology from the Seifert pairing}

We would like to relate the matrices $\mathcal{S}$ and $\overline{\mathcal{S}}$ to the longitudinal matrix $\left(c_{i j}^{S}\right)$ defined in Equation (3). For that we have to describe the procedure by which one defines a presentation for $H_{1}(\tilde{X}, \tilde{*})$, using a Seifert matrix. This will be entirely 
analogous to the traditional way of using a Seifert matrix of a knot or link $K$ to produce a presentation for $H_{1}\left(\widetilde{S^{3}-K}\right)$ (see $\left.[\mathrm{K}]\right)$.

Let $S, W, V$ be as above and let $Y=I \times D^{2}-V$. Let $i_{+}, i_{-}: V \rightarrow Y$ be defined by a push-off in the positive or negative normal direction, respectively. Now $V$ lifts into $\tilde{X}$ and the translates $\left\{V_{i}\right\}$ of $V$ cut $\tilde{X}$ into the union of the translates $\left\{Y_{i}\right\}$ of a lift of $Y$. We may WLOG move the base-point $*$ slightly into the interior of $Y$ and assume $* \in V$. Let $B$ be a small ball containing $*$ such that $B \cap V=B^{\prime}$ and $\partial B \cap Y=B_{+} \cup B_{-}$, where $B^{\prime}, B_{+}, B_{-}$are 2-disks. Now $\tilde{X}-\operatorname{int} \tilde{B}$ is a union of the $\left\{Y_{i}\right\}$ (now redefined as the lifts of $Y-(\operatorname{int} B \cap Y)$ ) attached along $\left\{V_{i}-B_{i}\right\}$, where the $\left\{B_{i}\right\}$ are the lifts of $B^{\prime}$.

A standard Mayer-Vietoris argument produces an exact sequence of $\mathbb{Z}\left[t, t^{-1}\right]$ modules:

$$
\mathbb{Z}\left[t, t^{-1}\right] \otimes H_{1}\left(V-B^{\prime}\right) \stackrel{\rho}{\rightarrow} \mathbb{Z}\left[t, t^{-1}\right] \otimes H_{1}\left(Y, B_{+} \cup B_{-}\right) \rightarrow H_{1}(\tilde{X}, \tilde{B}) \rightarrow 0
$$

where $\rho$ is the $\mathbb{Z}\left[t, t^{-1}\right]$-homomorphism defined by:

$$
\rho(1 \otimes \alpha)=t \otimes i_{+*}(\alpha)-1 \otimes i_{-*}(\alpha)
$$

Alexander duality gives us an isomorphism

$$
H_{1}\left(Y, B_{+} \cup B_{-}\right) \cong H^{1}\left(V-B^{\prime}, V \cap \partial\left(I \times D^{2}\right)\right)
$$

which is adjoint to the non-singular pairing:;

$$
\mathcal{L}: H_{1}\left(Y, B_{+} \cup B_{-}\right) \times H_{1}\left(V-B^{\prime}, V \cap \partial\left(I \times D^{2}\right)\right) \rightarrow \mathbb{Z}
$$

defined by linking number. Paths representing elements of these homology groups can be closed up (disjointly) in an obvious way.

From $\mathcal{L}$ we can define two Seifert pairings:

$$
\sigma_{ \pm}: H_{1}\left(V-B^{\prime}\right) \times H_{1}\left(V-B^{\prime}, V \cap \partial\left(I \times D^{2}\right)\right) \rightarrow \mathbb{Z}
$$

by $\sigma_{ \pm}(\alpha, \beta)=\mathcal{L}\left(i_{ \pm *}(\alpha), \beta\right)$. To get Seifert matrices we need to choose bases for $H_{1}\left(V-B^{\prime}\right)$ and $H_{1}\left(V-B^{\prime}, V \cap \partial\left(I \times D^{2}\right)\right)$. The basis for $H_{1}\left(V-B^{\prime}\right)$ can be the same basis we used for $H_{1}(V)$ in (18) with the addition of the class of $L_{m}$. For $H_{1}\left(V-B^{\prime}, V \cap \partial\left(I \times D^{2}\right)\right)$ we note that $V \cap \partial\left(I \times D^{2}\right)$ consists of $m$ disjoint arcs, one on each component of $\partial V=L_{S}$. Thus $H_{1}\left(V-B^{\prime}, V \cap \partial\left(I \times D^{2}\right)\right) \cong$ $H_{1}\left(V-B^{\prime}\right) \oplus \mathbb{Z}^{m-1}$. For basis we can choose the one already chosen for $H_{1}\left(V-B^{\prime}\right)$ together with the classes of the $\operatorname{arcs} a_{1}, \cdots, a_{m-1}$, where $a_{i}$ crosses the band $B_{i}$ connecting $L_{i} \cap \partial\left(I \times D^{2}\right)$ to $L_{i+1} \cap \partial\left(I \times D^{2}\right)$ (see Figure 4).

Let $A_{ \pm}$denote the matrix representing $\sigma_{ \pm}$with respect to these bases. So $t A_{+}-A_{-}$(with entries in $\mathbb{Z}\left[t, t^{-1}\right]$ ) represents the map $\rho$ in (24) and so is a presentation matrix of $H_{1}(\tilde{X}, \tilde{*})$. Note that $A_{ \pm}$has the form $A_{+}=\left(\begin{array}{ll}\tilde{A} & U_{+}\end{array}\right), A_{-}=$ ( $\tilde{A}^{t} U_{-}$), where $\tilde{A}$ is the matrix determined by $\bar{A}$ from (18) as follows:

$$
\tilde{A}=\left(\begin{array}{cc}
A & \tilde{B}^{t} \\
\tilde{B} & \Lambda
\end{array}\right)
$$




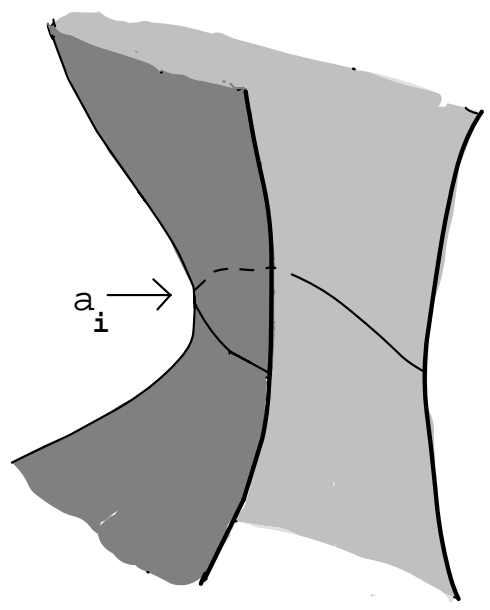

Figure 4

Definition of $a_{i}$

and $\tilde{B}$ is the matrix determined from $B$ by:

(i) The submatrix obtained by deleting the last row is $B$.

(ii) The sum of the rows of $\tilde{B}$ is 0 .

and $\Lambda$ is the matrix with entries $l_{i j}, 1 \leq i, j \leq m$ (see (19)). Assertion (ii) follows because $\sum_{i} L_{i}$ bounds $V$. $U_{ \pm}$is a matrix with $m-1$ columns whose entries are determined by the following values of the Seifert pairings:

$$
\begin{aligned}
\sigma_{ \pm}\left(\alpha, a_{j}\right)= & 0 \quad \text { if } \alpha \in H_{1}(W) \\
t_{i j}^{+}=\sigma_{+}\left(L_{i}, a_{j}\right) & = \begin{cases}0 & \text { if } j \text { is even } \\
1 & \text { if } i=j \text { is odd } \\
-1 & \text { if } i-1=j \text { is odd }\end{cases} \\
t_{i j}^{-}=\sigma_{-}\left(L_{i}, a_{j}\right) & = \begin{cases}0 & \text { if } j \text { is odd } \\
1 & \text { if } i=j \text { is even } \\
-1 & \text { if } i-1=j \text { is even }\end{cases}
\end{aligned}
$$

These assertions follow from the fact that $a_{j}$ bounds a small disk disjoint from $W$ and any $L_{i}$, for $i \neq j, j+1$ (see Figure 5 ).

Then we can write $U_{ \pm}=\left(\begin{array}{c}0 \\ T_{ \pm}\end{array}\right)$where $T_{ \pm}=\left(t_{i j}^{ \pm}\right)$is the $m \times(m-1)$-matrix defined in (27). 


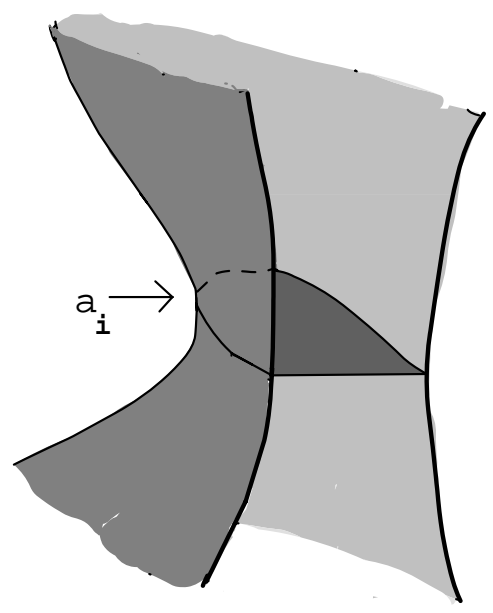

Figure 5 .

The disk bounded by $a_{i}$

Now the matrix $\mathcal{S}_{t}=t A-A^{t}$ is invertible in $\mathbb{Z}\left[t, t^{-1}\right]_{\Sigma}$ and so, by elementary row operations, we can convert the matrix $t A_{+}-A_{-}$to:

$$
\hat{\mathcal{S}}_{t}=\left(\begin{array}{ccc}
\mathcal{S}_{t} & (t-1) \tilde{B}^{t} & 0 \\
0 & (t-1) \Lambda-(t-1)^{2} \tilde{B} \mathcal{S}_{t}^{-1} \tilde{B}^{t} & t T_{+}-T_{-}
\end{array}\right)
$$

$\hat{\mathcal{S}}_{t}$ is now a presentation matrix for $H_{1}(\tilde{X}, \tilde{*})_{\Sigma}$ whose generators are the duals in $H_{1}\left(Y, B_{+} \cup B_{-}\right)$, with respect to the pairing $\mathcal{L}$, of the given basis $\left\{\beta_{i}, L_{i}, a_{i}\right\}$ of $H_{1}\left(V-B^{\prime}, V \cap \partial\left(I \times D^{2}\right)\right)\left(\beta_{i}\right.$ denotes a basis of $\left.H_{1}(W)\right)$. Let us consider the elements $\tilde{\lambda}_{i}, \mu_{i}^{\prime} \in H_{1}\left(Y, B_{+} \cup B_{-}\right)$, where $\tilde{\lambda}_{i}=i_{+*}\left(L_{i}\right)$, as in (3), and $\mu_{i}^{\prime}$ is the meridian curve which starts at $B_{+}$and travels along a positive push-off of a curve $\gamma_{i}$ in $W$ to $L_{i}$, half-way round a small meridian of $L_{i}$ and back to $B_{-}$along a negative push-off of $\gamma_{i}$ (see Figure 6).

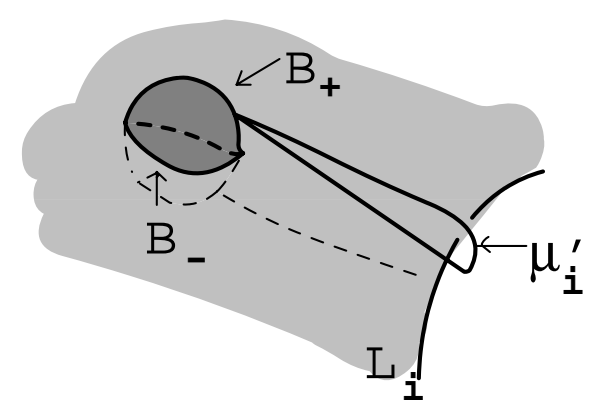

Figure 6 .

The meridian curve $\mu_{i}^{\prime}$ 
Then we have the following values of $\mathcal{L}$ :

$$
\begin{aligned}
\mathcal{L}\left(\tilde{\lambda}_{i}, L_{j}\right) & =l_{i j} \\
\mathcal{L}\left(\tilde{\lambda}_{i}, a_{j}\right) & =t_{i j}^{+} \\
\mathcal{L}\left(\tilde{\lambda}_{i}, \beta_{j}\right) & =\tilde{b}_{i j} \\
\mathcal{L}\left(\mu_{i}^{\prime}, L_{j}\right) & =\delta_{i j} \\
\mathcal{L}\left(\mu_{i}^{\prime}, a_{j}\right) & =0 \\
\mathcal{L}\left(\mu_{i}^{\prime}, \beta_{j}\right) & =0
\end{aligned}
$$

The last lines follows from the fact that $\gamma_{i}$ lies in $W$.

From (29) we can deduce $L_{i}^{\sharp}=\mu_{i}^{\prime}$ and:

$$
\tilde{\lambda}_{i}=\sum_{j} t_{i j}^{+} a_{j}^{\sharp}+\sum_{j} l_{i j} L_{j}^{\sharp}+\sum_{j} \tilde{b}_{i j} \beta_{j}^{\sharp}
$$

From the presentation matrix (28) we can write:

$$
\begin{array}{r}
\sum_{j} s_{i j} \beta_{j}^{\sharp}+(t-1) \sum_{j} \tilde{b}_{j i} L_{j}^{\sharp}=0 \\
(t-1) \sum_{j}\left(l_{i j}-(t-1) \theta_{i j}\right) L_{j}^{\sharp}+\sum_{j} \tau_{i j} a_{j}^{\sharp}=0
\end{array}
$$

where $\mathcal{S}_{t}=\left(s_{i j}\right), \tilde{B} \mathcal{S}_{t}^{-1} \tilde{B}^{t}=\left(\theta_{i j}\right)$ and $t T_{+}-T_{-}=\left(\tau_{i j}\right)$.

Note that $\mathcal{S}_{t}$ is invertible and we can see from $(27)$ that the $(m-1) \times(m-1)$ submatrix $\mathcal{T}$ obtained by deleting the last row of $t T_{+}-T_{-}$is invertible. Thus we may write:

$$
\begin{aligned}
& \beta_{i}^{\sharp}=(1-t) \sum_{r, j} \bar{s}_{i r} \tilde{b}_{j r} L_{j}^{\sharp} \\
& a_{i}^{\sharp}=(1-t) \sum_{r, j} \bar{\tau}_{i r}\left(l_{r j}-(t-1) \theta_{r j}\right) L_{j}^{\sharp}
\end{aligned}
$$

where $\mathcal{S}_{t}^{-1}=\left(\bar{s}_{i j}\right)$ and $\mathcal{T}^{-1}=\left(\bar{\tau}_{i j}\right)$ for $j \leq m-1$. For convenience later we define $\bar{\tau}_{i m}=0$ and let $\tilde{\mathcal{T}}_{t}=\left(\bar{\tau}_{i j}\right)$, for $j \leq m$.

Substituting (32) into (30) we obtain:

$$
\tilde{\lambda}_{i}=\sum_{j}\left((1-t) \sum_{s, r} t_{i s}^{+} \bar{\tau}_{s r}\left(l_{r j}-(t-1) \theta_{r j}\right)+l_{i j}+(1-t) \tilde{b}_{i s} \bar{s}_{s r} \tilde{b}_{j r}\right) L_{j}^{\sharp}
$$

Substituting the definition of $\theta_{i j}$ this becomes:

$$
\tilde{\lambda}_{i}=\sum_{j}\left((1-t) \sum_{s, r} t_{i s}^{+} \bar{\tau}_{s r}\left(l_{r j}-(t-1) \theta_{r j}\right)+l_{i j}-(t-1) \theta_{i j}\right) L_{j}^{\sharp}
$$


or, a bit more succinctly:

$$
\tilde{\lambda}_{i}=\sum_{j}\left(\sum_{r}\left(\delta_{i r}-(t-1) \sum_{s} t_{i s}^{+} \bar{\tau}_{s r}\right)\left(l_{r j}-(t-1) \theta_{r j}\right)\right) L_{j}^{\sharp}
$$

We can rewrite (34) briefly as:

$$
\tilde{\lambda}_{i}=\sum_{j} C_{i j} \mu_{j}^{\prime}
$$

where we define the matrix $C$ by:

$$
C=\left(I-(t-1) T_{+} \tilde{\mathcal{T}}_{t}\right)\left(\Lambda-(t-1) \tilde{B} \mathcal{S}_{t}^{-1} \tilde{B}^{t}\right)
$$

We now need to compare $C$ to $\left(c_{i j}^{S}\right)$ from (3). To do this we must compare the two sets of meridian generators $\left\{\tilde{\mu}_{i}\right\},\left\{\mu_{i}^{\prime}\right\}$ of $H_{1}(\tilde{X}, \tilde{*})$. The difference between the representative closed curves, $\left\{\tilde{u}_{i}\right\}$ and $\left\{u_{i}^{\prime}\right\}$, respectively, in $X$ is that the stems of $u_{i}$ lie in $0 \times D^{2}$ while the stems of $u_{i}^{\prime}$ lie essentially in $W$ (see Figure 7 ).

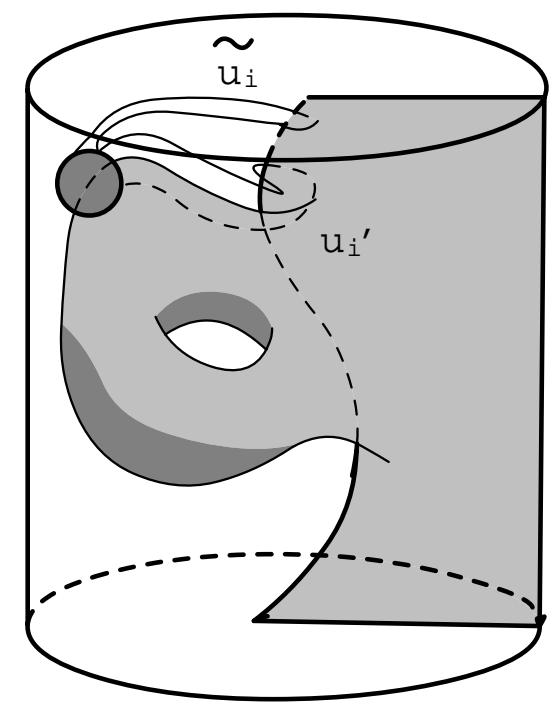

Figure 7 .

The two choices of meridian curves

However if we choose $W$ appropriately, then we can also choose $u_{i}^{\prime}$ to be a slight translate of $u_{i}$, since we have allowed ourselves to choose the stems of $u_{i}^{\prime}$ arbitrarily in $W$ (see Figure 8).

Thus we will have succeeded in showing that $C=\left(c_{i j}^{S}\right)$ (with the substitution $t=1+u)$ if we prove: 


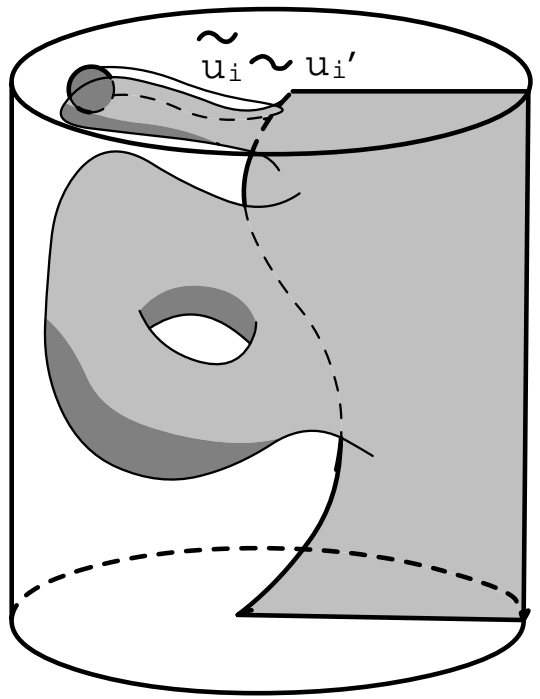

Figure 8.

How to make the two choices of meridian curves homotopic

Lemma 6.1. The matrix $\tilde{B} \mathcal{S}_{t}^{-1} \tilde{B}^{t}$ depends only on $S$, i.e. it is independent of the choice of $W$.

Proof. Any two choices of $W$ are cobordant so it follows from the usual argument (see e.g. [L]) that the Seifert matrices are S-equivalent. More precisely there are a sequence of elementary cobordisms connecting the two choices of $W$, where an elementary cobordism consists of adjoining or removing the boundary of a solid torus. The addition produces two new generators $\xi, \eta$ which are, respectively, the meridian and longitude curves of the torus. This changes $A$ by the following enlargement.

$$
A \longrightarrow\left(\begin{array}{ccc}
A & \vdots & 0 \\
\cdots & \cdot & 0 \\
0 & 1 & 0
\end{array}\right)
$$

where the last row and column corresponds to $\xi$. The effect on $\tilde{B}$ is the following enlargement.

$$
\tilde{B} \longrightarrow\left(\begin{array}{lll}
\tilde{B} & \cdot 0
\end{array}\right)
$$

From (37) we see that the resulting enlargement of $\mathcal{S}_{t}=t A-A^{t}$ is:

$$
\mathcal{S}_{t} \longrightarrow\left(\begin{array}{ccc}
\mathcal{S}_{t} & \vdots & 0 \\
\ldots & \cdot & -1 \\
0 & t & 0
\end{array}\right)
$$


From this we see that the resulting enlargement of $\mathcal{S}_{t}^{-1}$ is:

$$
\mathcal{S}_{t}^{-1} \longrightarrow\left(\begin{array}{ccc}
\mathcal{S}_{t}^{-1} & 0 & \vdots \\
0 & 0 & t^{-1} \\
\cdots & -1 & \cdot
\end{array}\right)
$$

Combining (38) and (39) and carrying out the matrix multiplication we can check that $\tilde{B} \mathcal{S}_{t}^{-1} \tilde{B}^{t}$ is unchanged by these enlargements of $\mathcal{S}_{t}^{-1}$ and $\tilde{B}$.

This completes the proof of Lemma 6.1.

\subsection{Completion of proof of Theorem 1}

We have now laid the necessary groundwork to relate the matrix $\Theta(z)=z \Lambda-$ $z^{2} B \mathcal{S}^{-1} B^{t}$ from (21), whose determinant is $\Gamma_{S}(z)$ (see $\left.(23)\right)$ and the matrix $\left(c_{i j}^{S}\right)$ from (3) which is a power series in $u=t-1$ whose coefficients are given, in Lemma 3.2 , in terms of the $\bar{\mu}$-invariants of $S$. Define $\tilde{\Theta}(z)=z \Lambda-z^{2} \tilde{B} \mathcal{S}^{-1} \tilde{B}^{t}$, so that $\Theta(z)$ is obtained from $\tilde{\Theta}(z)$ by deleting the last row and column, where $z=t-t^{-1}$. Then, using equation (36), we have:

$$
\begin{aligned}
\tilde{\Theta}(z) & =z\left(\Lambda-\left(t^{2}-1\right) \tilde{B} \mathcal{S}_{t^{2}}^{-1} \tilde{B}^{t}\right) \\
& =z\left(I-\left(t^{2}-1\right) T_{+} \tilde{\mathcal{T}}_{t^{2}}\right)^{-1}\left(c_{i j}^{S}(u)\right)
\end{aligned}
$$

where we are now using the substitution $u=t^{2}-1$. We now need to point out that $I-(t-1) T_{+} \tilde{\mathcal{T}}_{t}$ is a lower triangular matrix whose diagonal entries are $t^{-1}, 1, t^{-1}, 1, \ldots$. It is left to the reader to verify this from the definitions- see (27). From this we can now write:

$$
\operatorname{det} \Theta(z)=u^{m-1} t^{\epsilon} \operatorname{det}\left(c_{i j}^{S}\right)_{1 \leq i, j \leq m-1}
$$

where $\epsilon=\left\{\begin{array}{ll}0 & \text { if } m \text { is odd } \\ 1 & \text { if } m \text { is even }\end{array}\right.$ and $u=t^{2}-1=t z$. Substituting $t=\sqrt{u+1}$ and $z=u / \sqrt{u+1}$, this completes the proof of Theorem 1 .

\subsection{Proof of Corollary 1.3}

The proof begins with the argument on page 12 in the proof of Corollary 1.2. Thus we may assume that there are concordant string links $S, S^{\prime}$ whose closures are $L, L^{\prime}$, respectively. It follows immediately from their definitions, that $K_{S}$ and $K_{S^{\prime}}$ are concordant and, by the classical result of Fox-Milnor $[\mathrm{FM}]$, there exist $f(z), g(z)$, as in the statement of Corollary 1.3 , such that $\nabla_{K_{S}}(z) f(z) f(-z)=$ $\nabla_{K_{S^{\prime}}}(z) g(z) g(-z)$. Since $\Gamma_{S}(z)=\Gamma_{S^{\prime}}(z)$, the Corollary follows from Theorem 1 . 


\section{Proof of Proposition 1.4}

From Equation $(23)$ we have that $\Gamma_{S}(z)=(-1)^{m-1} z^{2(m-1)} \Phi(z)$ where $\Phi(z)=$ $\operatorname{det} A(t)$ and $A(t)$ is the skew-Hermitian matrix $B \mathcal{S}^{-1} B^{t}$. In this context $A(t)$ has entries in the localized ring $\mathbb{Z}\left[t, t^{-1}\right]_{\Sigma}$ and skew-Hermitian means the equality $A\left(t^{-1}\right)=-A(t)^{t}$. Of course $z=t-t^{-1}$ as usual. It follows immediately from the skew-Hermitian property that $\Phi(z)=(-1)^{m-1} \Phi(-z)$ which proves Assertion (1) in Proposition 1.4.

To prove (2) we place ourselves in the somewhat larger ring $\Lambda=\mathbb{Q}\left[t, t^{-1}\right]_{I}$, the localization at the principal ideal $I=(t-1) . \Lambda$ is a discrete valuation ring, i.e. all its ideals are powers of $I$ (or rather the ideal generated by $I$ in $\Lambda$ ). For such a ring it is a standard argument to show that the skew-Hermitian matrix $A(t)$ is congruent to a matrix $D(t)$ which is a block sum of $1 \times 1$-matrices $(\phi(t))$ and $2 \times 2$-matrices of the form $\left(\begin{array}{cc}0 & \psi(t) \\ -\psi\left(t^{-1}\right) & 0\end{array}\right)$. It follows that $\phi\left(t^{-1}\right)=-\phi(t)$, and so we can write $\phi(t)=\left(t-t^{-1}\right) \phi^{\prime}(t)$, where $\phi^{\prime}\left(t^{-1}\right)=\phi^{\prime}(t)$. Now we have $\Phi(t)=h(t) h\left(t^{-1}\right) \operatorname{det} D(t)$. If $D(t)$ has any block summands $(\phi(t))$, then $\Phi(t)$ is divisible by $t-t^{-1}$. If $m-1$ is even, then we must have another such summand and so $\Phi(t)$ is divisible by $\left(t-t^{-1}\right)^{2}$. In other words $\Phi(z)$ is divisible by $z^{2}$. If there are no such summands then it follows that $\Phi(t)$ must be of the form $g(t) g\left(t^{-1}\right)$. Since all the elements of $\Lambda$ are rational functions the proof is complete.

\section{Example}

To illustrate Theorem 1 we consider a very simple example. Let $S$ be the string link in Figure 9.

An easy computation gives:

$$
\lambda_{1}=\mu_{1}^{-1} \mu_{2} \quad \lambda_{2}=\mu_{2}^{-1} \mu_{1}
$$

The $\bar{\mu}$-invariants we need are computed easily from (41) and are given by:

$$
\mu_{i_{1}, \cdots, \subset_{r}, 1,1}=\left\{\begin{array}{l}
(-1)^{r+1} \text { if } i_{1}=\cdots=i_{r}=1 \\
0 \quad \text { otherwise }
\end{array}\right.
$$

Thus we see that $\lambda_{11}(u)=-u / u+1$, where $\lambda_{i j}(u)$ is defined in Theorem 1 . From the definition in Theorem 1 we see that

$$
\Gamma_{S}(z)=\sqrt{u+1}(-u / u+1)=-u / \sqrt{u+1}=-z
$$

Since $K_{S}$ is trivial, Theorem 1 tells us that $\nabla_{L_{S}}(z)=-z$, which checks out since $L_{S}$ is just the right-hand Hopf link. 


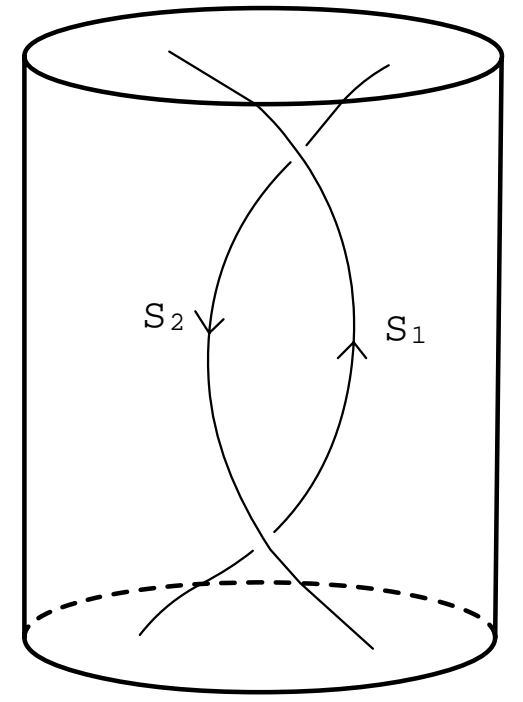

Figure 9.

A simple example

\section{References}

[C] T. Cochran, Concordance invariance of coefficients of Conway's link polynomial , Inventiones Math. 82 (1985), 527-541.

[FM] R. Fox and J. Milnor, Singularities of 2-spheres in 4-space and cobordism of knots, Osaka J. Math. 3 (1966), 257-67.

[HL] N. Habegger, X. S. Lin, The classification of links up to link homotopy, J. Amer. Math. Society 3 (1990), 389-419.

[HM] N. Habegger, G. Masbaum, The Kontsevich integral and Milnor's invariants, Preprint.

[H] J. Hoste, The first coefficient of the Conway polynomial, Proc. Amer. Math. Soc. 95 (1985), 299-302.

[K] L. Kauffman, On knots, Annals of Math. Studies 115, Princeton U. Press 1987.

[Ka] A. Kawauchi, On the Alexander polynomials of cobordant links, Osaka J. Math. 15 (1978), 151-159.

[L] J. Levine, Algebraic classification of some knots of codimension two, Comm. Math. Helv. 45 (1970), 185-198.

[L1] J. Levine, Symmetric presentation of link modules, Topology and its Applic. 30 (1988), 183-198.

[L2] J. Levine, The $\bar{\mu}$-invariants of based links, Differential Topology, Proceedings Siegen 1987 (ed. U. Koschorke), Lecture Notes 1350, Springer-Verlag, New York, 87-103.

[L3] J. Levine, The Conway polynomial of an algebraically split link, Proceedings KNOTS96 (to appear).

[M] W. Magnus, Beziehungen zwischen Gruppen und Idealen in einem speziellen Ring, Math. Annalen 111 (1935), 259-280.

[Mi] J. Milnor, Isotopy of links, Algebraic Geometry and Topology: a symposium in honor of Solomon Lefschetz, Princeton, NJ 1957.

[N] Y. Nakagawa, On the Alexander polynomials of slice links, Osaka J. Math. 15 (1978), 
$161-182$.

[R] L. Rozansky, A contribution of the trivial connection to the Jones polynomial and Witten's invariant of 3d manifolds II, Comm. in Math. Physics 175 (1996), 297-318.

[S] J. Stallings, Homology and central series of groups, Journal of Algebra 2 (1965), 170-181.

[T] L. Traldi, Milnor's invariants and the completions of link modules, Trans. Amer. Math. Soc. 284 (1984), 401-424.

[T1] L. Traldi, Conway's potential function and its Taylor series, Kobe J. Math. 5 (1988), 233-264.

[Tr] A. Tristram, Some cobordism invariants for links, Proc. Cambridge Phil. Soc. 66 (1969), 251-264.

Jerome Levine

Department of Mathematics

Brandeis University

Waltham, MA 02254-9110, USA

e-mail: levine@binah.cc.brandeis.edu

(Received: April 12, 1997) 